\title{
Design and Implementation of a High Voltage Source for Biphasic Electrical Stimulators
}

\author{
Hasan H. Eroglu \\ Department of Electrical and Electronics Engineering \\ Bartın University \\ Bartın, Türkiye \\ heroglu@bartin.edu.tr
}

\begin{abstract}
Biphasic constant current (CC) stimulators are used in functional electrical stimulation applications. These stimulators require positive and negative high voltage (HV) sources in order to deliver the programmed CC pulses to load. For example, applying symmetrical biphasic CC pulses with $50 \mathrm{~mA}$ amplitude to a $1 \mathrm{k} \Omega$ load requires positive and negative supply rails of $50 \mathrm{~V}$ and $-50 \mathrm{~V}$, respectively. In this study, a HV source based on a switched inductance boost DC/DC converter and a switched capacitor voltage inverter is designed and implemented in order to satisfy the $\mathrm{HV}$ requirements of biphasic stimulators. Performance of the proposed HV topology is satisfactory for CC pulses of $35 \mathrm{~mA}$ applied to a $1 \mathrm{k} \Omega$ load.
\end{abstract}

Keywords-biphasic current source; high voltage; switched inductor; switched capacitor; DC/DC converter

\section{INTRODUCTION}

Functional electrical stimulation (FES) is a technique based on injecting electrical current pulses to excitable biological tissue. A bodily function is restored in patients with neurological disorders. Foot drop correction, hand grasping and releasing control, bladder and bowel control, standing assistance, cough and breathing assistance are some of the FES applications [1]. In FES applications, electrical stimulators are used for delivering constant voltage (CV) or constant current (CC) pulses to body. CC stimulators are used in many applications, where stable physiological response is required since $\mathrm{CC}$ stimulators result more stable response in terms of muscle contraction consistency and repeatability compared to CV stimulators. Biphasic current is preferred in many $\mathrm{CC}$ stimulation applications since it reduces charge accumulation risk by providing bidirectional ion flow [2].

Biphasic CC stimulators should satisfy output voltage requirements of the load. For example, applying a symmetrical biphasic current waveform with $50 \mathrm{~mA}$ amplitude to a $1 \mathrm{k} \Omega$ load requires positive $\left(V_{H V}^{+}\right)$and negative $\left(V_{H V}^{-}\right)$high voltage (HV) levels of $50 \mathrm{~V}$ and $-50 \mathrm{~V}$, respectively. Ilic et al. used a multi-output flyback DC/DC converter to provide $V_{H V}^{+}$and $V_{H V}^{-}$[3]. The flyback converter isolates the output stage with low voltage electronics. However, utilization of the flyback transformer increases device size and may cause electromagnetic interference. Non-isolated switched inductance (SI) boost and buck-boost DC/DC converters can be utilized for generating $V_{H V}^{+}$and $V_{H V}^{-}$, respectively [4], [5]. Recently, Karpul et al. expressed that boost converters are noisy and difficult to implement. They proposed utilization of cascade- connected commercially available switched capacitor (SC) converters to supply $V_{H V}^{+}$and $V_{H V}^{-}$[6].

In this study, it is aimed to design and implement a HV source for biphasic CC stimulators. The proposed HV source is a hybrid topology based on conventional boost $\mathrm{DC} / \mathrm{DC}$ converter and $\mathrm{SC}$ voltage inverter. Physical realization of the proposed HV source is documented and performance of the proposed topology is evaluated by using physical experiments.

\section{A BIPHASIC CC SOURCE}

A biphasic CC source is considered as shown in Fig. 1 [2], [5]. Output current $\left(i_{\text {out }}\right)$ is adjusted by using set voltage $\left(v_{\text {set }}\right)$ and resistors $\left(R_{\text {set } 1}, R_{\text {set } 2}\right)$. Operational amplifier 1 (OP1), bipolar junction transistor (BJT) 1 (Q1), $R_{\text {set } 1}$ and OP2, Q5, $R_{\text {set } 2}$ adjust positive $\left(i_{p}\right)$ and negative output current $\left(i_{n}\right)$, which are applied to load resistance $\left(R_{\text {load }}\right)$ through sourcing and sinking current mirrors composed of Q2, Q3, Q4 and Q6, Q7, Q8, respectively. Stabilizing resistors $\left(R_{s t b}\right)$ are used to provide $i_{\text {out }}$ insensitive to base emitter voltages of the BJTs [2]. $i_{p}$ and $i_{n}$ can be expressed as

$$
i_{p}=\frac{v_{\text {set }}}{R_{\text {set } 1}} \leq \frac{V_{H V^{+}}-2 V_{C E-s a t}}{R_{\text {load }}+R_{\text {stb }}}
$$

and

$$
i_{n}=\frac{v_{\text {set }}}{R_{\text {set } 2}} \leq \frac{V_{H V^{-}}-2 V_{C E-s a t}}{R_{\text {load }}+R_{\text {stb }}}
$$

where $V_{C E-s a t}$ is collector emitter saturation voltage of the BJTs.

\section{PROPOSED HV SOURCE AND ITS CONTROL}

Proposed HV source for satisfying $V_{H V}^{+}$and $V_{H V}^{-}$ requirements of biphasic $\mathrm{CC}$ stimulators is composed of an SI boost DC/DC converter and an SC voltage inverter as shown in Fig.2. The boost converter is an SI converter used for stepping-up input voltage $\left(V_{i n}\right)$ [7]. As shown in Fig. 2 (a), the boost converter is composed of an inductor $(L)$, a controllable switch $(S)$, a diode (D1) and an output capacitor $\left(C_{\text {out } 1}\right)$. Output voltage ( $\left.V_{\text {out }}\right)$ of the boost converter $\left(V_{H V}^{+}\right)$can be controlled through pulse width modulation (PWM). When the PWM signal is high, energy is stored in $L$ through $V_{\text {in }}$ and $S$. The energy stored in $L$ is transferred to $C_{\text {out } 1}$ through $D_{1}$ as the PWM signal is low. In electrical stimulation applications, boost converters operate in discontinuous conduction mode (DCM) since ripple component of inductor current $\left(\Delta i_{L}\right)$ is generally greater 


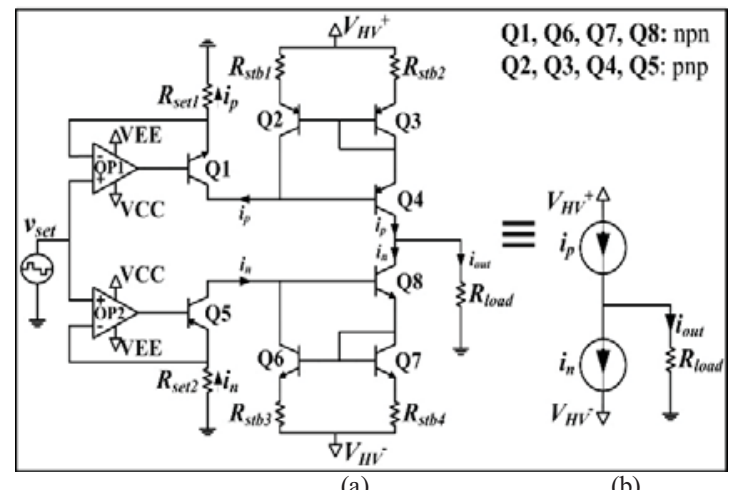

(a)

(b)

Figure 1. A biphasic CC source (a) and its equivalent circuit (b).

than its DC component $\left(I_{L}\right)$ due to low $i_{\text {out }}(\sim 1-100$ $\mathrm{mA})$ and high $V_{\text {out }}(\sim 100 \mathrm{~V})$ requirements. Voltage conversion ratio of the boost converter $\left(A_{V}\right)$ in DCM can be expressed as

$$
A_{V}=\frac{V_{\text {out }}}{V_{\text {in }}}=\frac{1+\sqrt{1+2 D^{2} R_{\text {load }} T_{S} / L}}{2}
$$

where $D$ and $T_{S}$ are duty cycle and period of the PWM signal, respectively [7].

Hybrid converters composed of SI and SC converters are used in industrial applications, such as power sources of liquid crystal displays (LCD) [8]. By utilizing a similar perspective, $V_{H V}^{-}$requirement of the biphasic stimulator can also be satisfied with an SC inverter connected to the switching node $(A)$ of the boost converter. By using this topology, $V_{H V}^{+}$and $V_{H V}^{-}$ requirements of the biphasic stimulator can be satisfied with a single controllable switch $(S)$ and an inductor $(L)$. As shown in Fig. 2 (a), the SC inverter is composed of diodes D2, D3, pump $\left(C_{p}\right)$ and output $\left(C_{\text {out } 2}\right)$ capacitors. Switching signal required at input of the SC inverter is supplied by voltage on $A\left(V_{A}\right)$. When $V_{A}$ is high $\left(V_{A}=V_{H V}^{+}\right), D_{2}$ and $D_{3}$ are forward and reverse biased, respectively. $C_{p}$ is charged through $D_{2}$ to $V_{H V^{+}}-V_{f w}$, where $V_{f w}$ is the forward voltage drop of the diodes. When $V_{A}$ is low $\left(V_{A}=0\right)$, the voltage on $B$ is equal to $-\left(V_{i n}-V_{f w}\right)$ due to the voltage on $C_{p}$. As a result, $D_{3}$ is forward biased and $C_{\text {out2 }}$ is charged through $D_{3}$ to $-\left(V_{i n}-2 V_{f w}\right)$. For continuous operation of $S, V_{H V}^{-}$can be expressed as

$$
V_{H V}^{-}=-\left[V_{H V}^{+}-2 V_{f w}-i_{n} /\left(f C_{p}\right)\right],
$$

where $f$ is switching frequency and $1 /\left(f C_{p}\right)$ is output impedance of the SC inverter.

Output voltages of the proposed HV topology are controlled using the process shown in Fig. 2 (b). $V_{H V^{+}}$ and $V_{H V^{-}}$are measured as $V_{m 1}$ and $V_{m 2}$ by using resistor dividers composed of $R_{m 1}$ and $R_{m 2} \cdot V_{m 2}$ is inverted by an inverting amplifier $\left(V_{m 2}^{\prime}=-V_{m 2}\right)$. $V_{m 1}$ and $V_{m 2}^{\prime}$ are compared with a voltage reference $\left(V_{\text {ref }}\right)$ obtained from a resistor-capacitor $\left(R_{s t}, C_{s t}\right)$ soft start circuit. Comparator outputs are buffered and fed into a NOR gate in order to generate a reset signal $(R)$, which is active when both $V_{m 1}$ and $V_{m 2}^{\prime}$ are above $V_{\text {ref }}$. In the next step, $R$ and a PWM signal are fed to a set-reset (SR) latch which is used to control the gate driver.

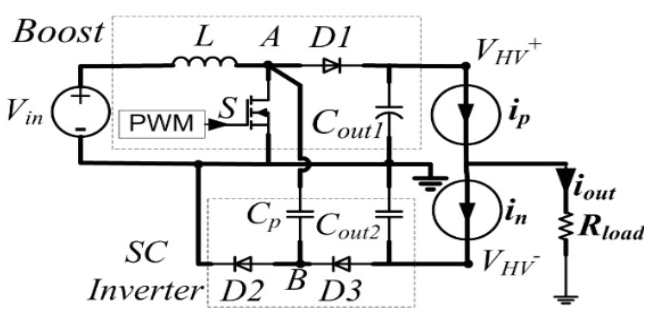

(a)

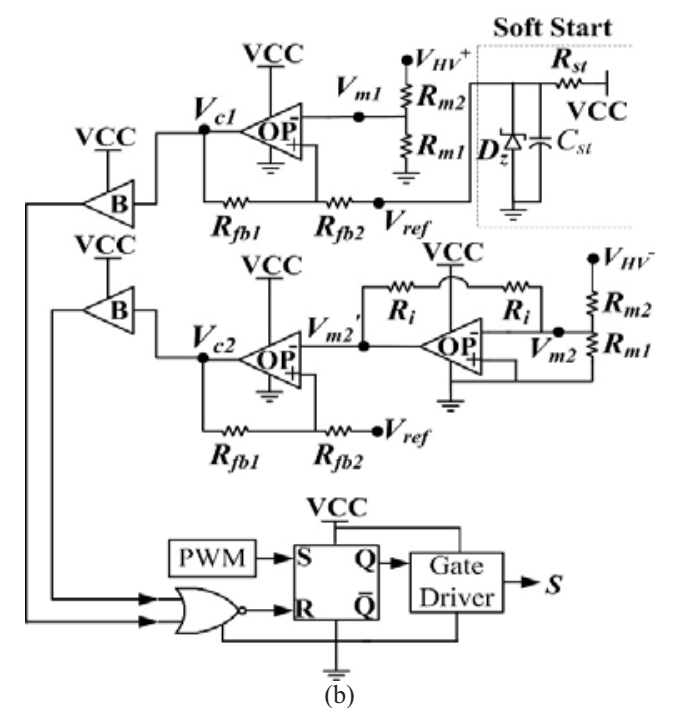

Figure 2. Proposed HV source for biphasic CC stimulators (a) and its output voltage control process (b)

Start-up procedure of the proposed topology can be explained as follows. No-load condition is considered. Initially, $V_{r e f}$ is zero due to the soft start circuit. $V_{i n}$ charges $C_{\text {out } 1}$ to $V_{\text {in }}-V_{f w}$ immediately. $C_{\text {out } 2}$ is also charged to a very low level. Therefore, initially, both $V_{m 1}$ and $V_{m 2}{ }^{\prime}$ are above $V_{\text {ref }}$ and the switching signal $(S)$ of the gate driver is zero. As $V_{\text {ref }}$ reaches $V_{m 2}{ }^{\prime}, S$ becomes active, both $V_{m 1}$ and $V_{m 2}$ ' (equivalently $V_{H V}^{+}$ and $V_{H V}^{-}$) increases. During the start-up, $V_{m 2}{ }^{\prime}$ tracks $V_{\text {ref }}$. On the other hand, $V_{m 1}$ overshoots $V_{\text {ref }}$ since $V_{\text {ref }}$ reaches $V_{m 2}{ }^{\prime}$ at first. However, both $V_{m 1}$ and $V_{m 2}{ }^{\prime}$ increase in similar rates if $C_{\text {out } 1}$ and $C_{\text {out } 2}$ are chosen equally. In biphasic stimulation applications, $i_{p}$ and $i_{n}$ are supplied by $V_{H V}^{+}$and $V_{H V}^{-}$, respectively. During the application of $i_{n}$ to $R_{\text {load }}, S$ regulates $V_{m 2}$ ' (equivalently $V_{H V}^{-}$) to $V_{\text {ref }}$. During this period, $V_{m 1}$ (equivalently $V_{H V}^{+}$) also increases and overshoots $V_{\text {ref }}$. When $i_{p}$ is applied to $R_{\text {load }}, V_{m 1}$ decreases through $V_{\text {ref }}$ and $S$ regulates $V_{m 1}$ as soon as $V_{m 1}$ reaches $V_{\text {ref }}$.

\section{Physical REALIZATION}

The proposed HV source is implemented as a printed circuit board. Properties of the HV source are summarized in Table I. A biphasic CC source based on the circuit in Fig. 1 is used as the load of the HV source. The biphasic CC source is controlled by a signal generator composed of a microcontroller and a digital to analog converter as explained in [5]. A resistive load of $1 \mathrm{k} \Omega$ is used at the output of the biphasic CC source. Experimental set-up is shown in Fig.3. The HV source 
TABLE I. Properties of the Implemented HV Source

\begin{tabular}{ll}
\hline Component & Part Name and/or Properties \\
\hline VCC $\left(V_{\text {in }}\right)$ & $12 \mathrm{~V}$ \\
\hline$S$ & IRF530, n-channel MOSFET \\
\hline$L 1, D 2, D 3$ & SB560, Schottky Diode \\
\hline$C_{\text {out } 1}, C_{\text {out } 2}$ & ABCO, $10 \mu \mathrm{H}, 1 \mathrm{~A}$ \\
\hline$C_{p}$ & Wecon, $63 \mathrm{~V}, 47 \mu \mathrm{F}$ electrolytic $, 63 \mathrm{~V}, 680 \mathrm{nF}$ polyester \\
\hline NOR gate & RTL logic, $10 \mathrm{k} \Omega$, BC237 \\
\hline Gate Driver & IR2153 Self Oscillating Gate Driver, $f=180 \mathrm{kHz}$ \\
\hline OP & LM324, quadruple operational amplifier \\
\hline$R_{m 1}, R_{m 2}, R_{i}$ & $1 \mathrm{M} \Omega, 10 \mathrm{M} \Omega, 1 \mathrm{M} \Omega$ \\
\hline Soft Start $\left(D_{z}\right)$ & Zener Diode, $V_{\text {break }}=3.2 \mathrm{~V}$ \\
\hline Soft Start $(R, C)$ & $10 \mathrm{k} \Omega, 47 \mu \mathrm{F}$ \\
\hline
\end{tabular}

is supplied by a $12 \mathrm{~V}$ DC power source. Quiescent current of the HV source is $40 \mathrm{~mA}$. Transient and steady-state characteristics of the HV source are measured by using a 2-channel oscilloscope with 100 $\mathrm{MHz}$ bandwidth (AA Tech ADS3202B).

\section{EXPERIMENTAL RESULTS}

The implemented HV source is turned on for no load condition. Oscillograms for transient response of $V_{m 1}, V_{m 2}^{\prime}, V_{H V}^{+}$and $V_{H V}^{-}$are shown in Fig. 4. As shown in Fig. 4, $V_{m 1}$ and $V_{m 2}^{\prime}$ increase suddenly as $V_{i n}$ is applied. $V_{m 2}^{\prime}$ tracks $V_{\text {ref }}$ as soon as $V_{\text {ref }}$ reaches $V_{m 2}^{\prime}$. $V_{m 1}$ (equivalently $V_{H V}^{+}$) is relatively constant when $V_{m 2}^{\prime}$ is smaller than the initial level of $V_{m 1}$ during start-up. During this stage, the stored energy in $L$ is transferred to the SC inverter since $D 1$ is reverse biased. As $V_{m 2}^{\prime}$ reaches $V_{m 1}$, both $V_{m 1}$ and $V_{m 2}^{\prime}$ (equivalently $V_{H V}^{+}$and $\left.V_{H V}^{-}\right)$reach steady-state. It is observed that both $V_{H V}^{+}$ and $V_{H V}^{-}$reach the steady-state values of $38.4 \mathrm{~V}$ and $39.2 \mathrm{~V}$ in a $500 \mathrm{~ms}$ time period.

Amplitude and frequency of the biphasic $\mathrm{CC}$ source are adjusted as $35 \mathrm{~mA}$ and $50 \mathrm{~Hz}$. In order to observe the steady-state characteristics of the proposed HV source for different CC load conditions, the pulse widths of $i_{p}$ and $i_{n}$ are adjusted as $1 \mathrm{~ms}, 3 \mathrm{~ms}$, and 10 $\mathrm{ms}$. Oscillograms for the steady-state response of the HV source for different $\mathrm{CC}$ loads are shown in Fig. 5. As shown in Fig. $5, \pm 35 \mathrm{~V}$ is observed on the $1 \mathrm{k} \Omega$ load due to the $i_{p}$ and $i_{n}$ pulses with $35 \mathrm{~mA}$ amplitude. As shown Fig. 5 (b, d, f), $i_{n}$ pulses discharge $C_{\text {out } 2}$ which decreases $V_{H V}^{-}$. The controller drives $S$ in order to regulate $V_{H V}^{-}$. During the period in which $S$ regulates $V_{H V}^{-}, V_{H V}^{+}$also increases and overshoots the reference level, as shown Fig. 5 (a, c, e). The additional energy stored in $C_{\text {out } 1}$ during the overshoot periods are dissipated by the $i_{p}$ pulses. The average $V_{H V}^{+}$values are measured as $38.8 \mathrm{~V}, 38 \mathrm{~V}$, and $38 \mathrm{~V}$ for the $i_{p}$ and $i_{n}$ pulse widths of $1 \mathrm{~ms}, 3 \mathrm{~ms}$, and $10 \mathrm{~ms}$, respectively. The corresponding average $V_{H V}^{-}$values are $39.2 \mathrm{~V}$, $39.6 \mathrm{~V}$, and $37 \mathrm{~V}$, respectively. It is seen that the proposed HV source can supply $i_{p}$ and $i_{n}$ pulses with $35 \mathrm{~mA}$ amplitude and different pulse widths.

\section{DISCUSSION AND CONCLUSIONS}

A HV source satisfying the requirements of a biphasic CC stimulator is designed and implemented. $V_{H V}^{+}$is supplied by the SI boost converter with 4 basic components $\left(L, S, D_{1}, C_{\text {out } 1}\right)$. The boost converter with

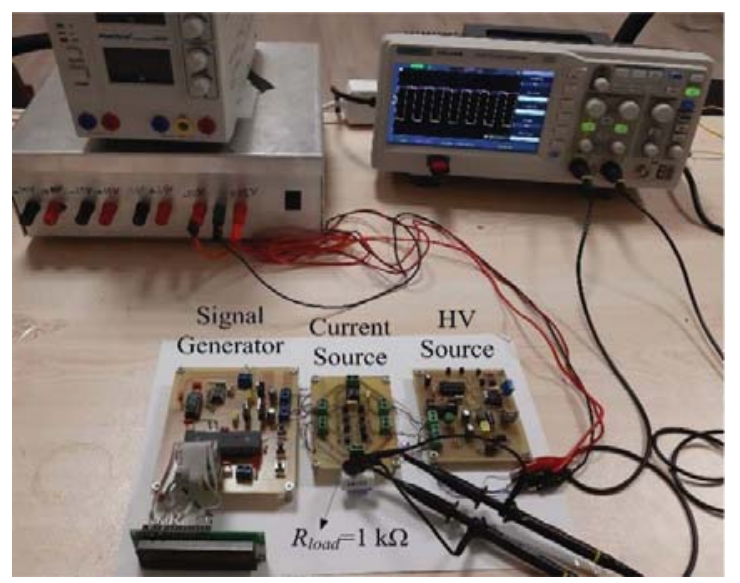

Figure 3. The experimental set-up.

minimum number of components is very easy to implement. $V_{H V}^{-}$is easily obtained by adding two diodes and two capacitors $\left(D 2, D 3, C_{p}, C_{\text {out } 2}\right)$ to the switching node of the boost converter. By using a relatively high switching frequency of $180 \mathrm{kHz}$, the boost converter is realized with a small inductance of $10 \mu \mathrm{H}$. Size of the proposed HV source is kept small (i.e. $75 \times 88 \mathrm{~mm}^{2}$ ) although through-hole discrete components are used as shown in Table I.

In desktop and portable applications, the HV source can be supplied by a medical grade power source and a battery, respectively. Excluding the inductor and capacitors, the HV source can be implemented as an integrated circuit. Steady-state $V_{H V}^{+}$and $V_{H V}^{-}$ measurements are between $37 \mathrm{~V}$ and $39 \mathrm{~V}$ for different conditions. Using the HV source, shaped bipolar current pulses between $37 \mathrm{~mA}$ and $-39 \mathrm{~mA}$ can be supplied to a $1 \mathrm{k} \Omega$ load. $i_{\text {out }}$ rating can be improved by adjusting $V_{H V}^{+}$and $V_{H V}^{-}$through $V_{r e f}, R_{m 1}$, and $R_{m 2}$ As expressed in [4] and [5], $12 \mathrm{~V}$ to $100 \mathrm{~V}$ conversion can be obtained with the proposed single stage SI boost converter. Larger voltage swing in the order of $300 \mathrm{~V}$ can be achieved with two-stage SI boost converters [4]. The proposed HV topology provides regulated $v_{\text {out }}$ for different $i_{\text {out }}$ conditions, whereas $v_{\text {out }}$ of the cascaded SC inverter solution in [6] will decrease as amplitude and duty cycle of $i_{\text {out }}$ increases due to the output impedance of the inverters. The HV solution in [6] is evaluated for a low $i_{\text {out }}$ level of $1 \mathrm{~mA}$ which is much smaller than the $i_{\text {out }}$ level of $35 \mathrm{~mA}$ used in this study. The proposed HV topology is a design competitive low-cost solution for commercial stimulators with large currents.

As shown in Fig.5, $V_{m 2}^{\prime}$ tracks $V_{\text {ref }}$ and $V_{m 1}$ overshoots $V_{\text {ref }}$ during the operation of the HV source. The overshoot in $V_{m 1}$ can be eliminated by using a chopper composed of a resistor and a ground referenced switch in parallel with $C_{\text {out } 1}$. In this configuration, the chopper will remove the additional energy stored in $C_{\text {out } 1}$, when $V_{m 1}$ overshoots $v_{\text {ref }}$. Designers can also add a logic to the system to block $S$ if $V_{m 1}$ reaches a certain threshold, as a potential safety measure. Output voltage tracking characteristics of the 

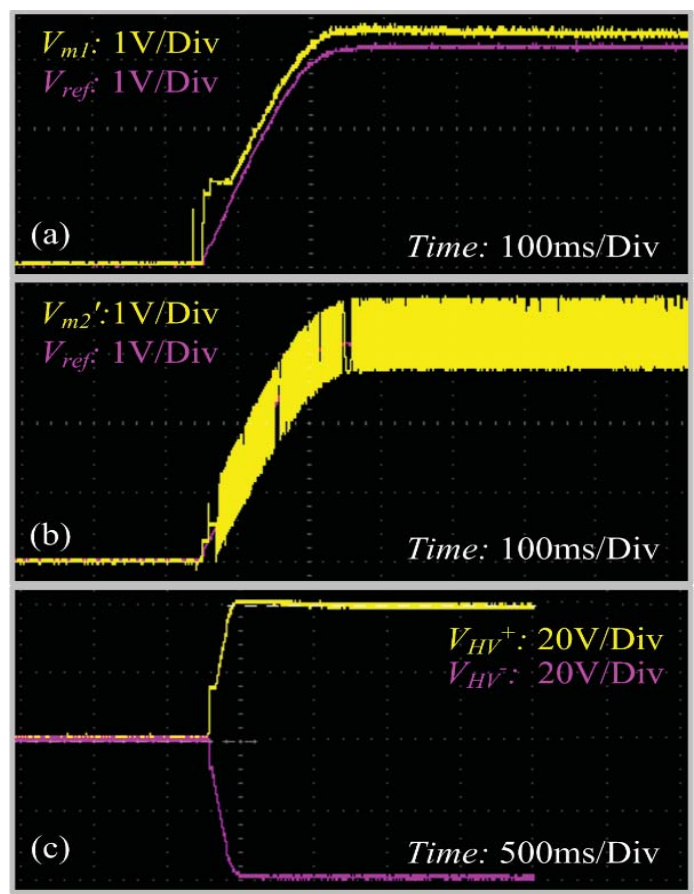

Figure 4. Oscillograms obtained for the transient response of $V_{m 1}$ (a), $V_{m 2}^{\prime}$ (b), $V_{H V}^{+}$and $V_{H V}^{-}$(c).

proposed HV source can be changed by applying a different logic design in the control block shown in Fig 2 (b). For example, if a NAND gate is used instead of the NOR gate, $V_{m 1}$ will track $v_{r e f}$. For the control block with a NAND gate, the turn-on process of the HV source can be explained as follows. At first, $V_{m 1}$ and $V_{m 2}^{\prime}$ increase suddenly as $V_{i n}$ is applied. $S$ is zero since $V_{\text {ref }}$ is below $V_{m 1}$ and $V_{m 2}^{\prime}$. As $V_{\text {ref }}$ reaches $V_{m 2}^{\prime}, S$ remains zero since $V_{\text {ref }}$ is still below $V_{m 1}$. As $V_{\text {ref }}$ reaches $V_{m 1}, S$ becomes active and regulates $V_{m 1}$ to track $V_{\text {ref }} . V_{m 2}^{\prime}$ also increases during the period in which $S$ is active. In the steady-state, $V_{m 1}$ tracks $V_{\text {ref }}$, whereas $V_{m 2}^{\prime}$ is below $V_{\text {ref }}$. During the $i_{p}$ pulses, $S$ becomes active and regulates $V_{m 1}$, which also increases $V_{m 2}^{\prime}$. However, $S$ is not active during the $i_{n}$ pulses which decreases $V_{m 2}^{\prime}$. It is observed that the control block regulates the measured voltage which $V_{\text {ref }}$ reaches first. As shown in Fig. 5, $C_{\text {out } 1}$ and $C_{\text {out } 2}$ filter out the high frequency switching noise in the steady steady-state $V_{H V}^{+}$and $V_{H V}^{-}$waveforms. Therefore, the noise is not observed as a problem for the SI boost converter and SC inverter which is mentioned in [6].

$V_{H V}^{-}$can be obtained by a using an SI buck-boost converter with inverted output [5]. However, utilizing a buck-boost converter has several disadvantages. The first one is to use an additional inductor which will increase the size of the HV source. The second disadvantage is the need for a high side controllable switch. Since there is a single input voltage source, an isolated gate driver for the high side switch is not an option. Standard half bridge MOSFET gate drivers with bootstrap function are not suitable for the high side switches of the buck boost converters. Using a p-channel MOSFET seems to be the only solution. As the third disadvantage, the buck-boost topology also requires a separate output voltage control block, an oscillator and a gate driver.

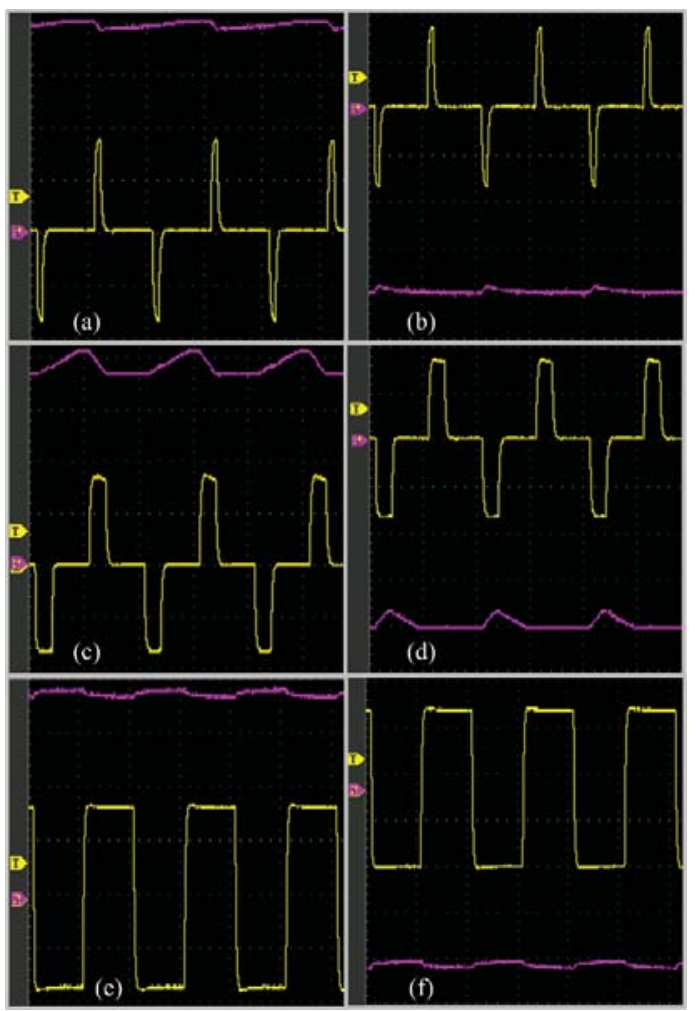

Figure 5. Oscillograms obtained for the steady-state response of $V_{H V}^{+}(\mathrm{a}, \mathrm{c}, \mathrm{e})$ and $V_{H V}^{-}(\mathrm{b}, \mathrm{d}, \mathrm{f})$ for $i_{p}$ and $i_{n}$ pulses with $35 \mathrm{~mA}$ amplitude and $1 \mathrm{~ms} \mathrm{(a,b),} 3 \mathrm{~ms}(\mathrm{c}, \mathrm{d})$, and $10 \mathrm{~ms}(\mathrm{e}, \mathrm{f})$ pulsewidths. Yellow waveforms represent $i_{\text {out }}$. Purple waveforms represent $V_{H V}^{+}$(a, c, e) and $V_{H V}^{-}(\mathrm{b}, \mathrm{d}, \mathrm{f})$. Voltage and time divisions are $20 \mathrm{~V} / \mathrm{div}$ and $10 \mathrm{~ms}$ for both channels.

Finally it can be concluded that the implemented HV source topology can be used in any application requiring bipolar voltages in addition to biphasic electrical stimulators.

\section{REFERENCES}

[1] J. O. Teeter, C. Kantor, and D. L. Brown, Functional Electrical Stimulation (FES) Resource Guide for Persons with Spinal Cord Injury or Multiple Sclerosis, Cleveland, Ohio: FES Information Center, 1995.

[2] H-C. Wu, S-T. Young, T-S. Kuo, "A versatile multichannel direct-synthesized electrical stimulator for FES applications," IEEE Trans. Inst. Meas., vol. 51, no. 1, pp. 2-9, 2002.

[3] M. Ilic, D. Vasiljevic, D. B. Popovic, "A programmable electronic stimulator for FES systems," IEEE Trans. Reh. Eng., vol. 2, no. 4, pp. 234-239, 1994

[4] N. Jorgovanovic, S. Dosen, R. Petrovic, "Novel electronic stimulator for functional electrical therapy," J. Aut. Cont. vol. 15, pp. 27-30, 2005.

[5] H. H. Eroglu, B. M. Eyuboglu, C. Goksu, "Design and implementation of a bipolar current source for MREIT applications," in Proc. XIII Medt. Conf. Med. Biol. Eng. Comp., Seville, Spain, 2013, pp. 161-164.

[6] D. Karpul, G. K. Cohen, G. D. Gargiulo, A. van Schaik, S. McIntyre, P. P. Breen, "Low-power transcutaneous current stimulator for wearable applications," Biomed. Eng. Onl. vol. 16, no. 1, (118), 2017.

[7] R. W. Erickson and D. Maksimovic, "Converter Circuits," in Fundamentals of Power Electronics, 2nd ed., New York: Kluwer Academic Publishers, 2004, pp. 131-184, 2010.

[8] S. Johnson, "Design for a Discrete Charge Pump," Texas Instruments Application Report, SLVA398A, 2010. 